BMJ Open

Diabetes

Research

\& Care

\section{Development of a multivariable prediction model for plantar foot ulcer recurrence in high-risk people with diabetes}

\author{
Wouter B aan de Stegge (D , , Ameen Abu-Hanna, ${ }^{2}$ Sicco A Bus (D) ${ }^{1}$
}

To cite: aan de Stegge WB, Abu-Hanna A, Bus SA. Development of a multivariable prediction model for plantar foot ulcer recurrence in highrisk people with diabetes. BMJ Open Diab Res Care 2020;8:e001207. doi:10.1136/ bmjdrc-2020-001207

Received 21 January 2020 Revised 17 February 2020 Accepted 26 February 2020

\section{Check for updates}

(c) Author(s) (or their employer(s)) 2020. Re-use permitted under CC BY. Published by BMJ.

${ }^{1}$ Department of Rehabilitation Medicine, University of Amsterdam, Amsterdam UMC, Amsterdam, The Netherlands ${ }^{2}$ Department of Medical Informatics, Universitiy of Amsterdam, Amsterdam UMC, Amsterdam, The Netherlands

Correspondence to Dr Sicco A Bus; s.a.bus@amsterdamumc.nl

\section{ABSTRACT}

Introduction Forty per cent of people with diabetes who heal from a foot ulcer recur within 1 year. The aim was to develop a prediction model for plantar foot ulcer recurrence and to validate its predictive performance. Research design and methods Data were retrieved from a prospective analysis of 171 high-risk patients with 18 months follow-up. Demographic, disease-related, biomechanical and behavioral factors were included as potential predictors. Two logistic regression models were created. Model 1 for all recurrent plantar foot ulcers $(71$ cases) and model 2 for those ulcers indicated to be the result of unrecognized repetitive stress (41 cases). Ten-fold cross-validation, each including five multiple imputation sets, was used to internally validate the prediction strategy; model performance was assessed in terms of discrimination and calibration.

Results The presence of a minor lesion, living alone, increased barefoot peak plantar pressure, longer duration of having a previous foot ulcer and less variation in daily stride count were predictors of the first model. The area under the receiver operating curve was 0.68 (IQR 0.610.80 ) and the Brier score was 0.24 (IQR 0.20-0.28). The predictors of the second model were presence of a minor lesion, longer duration of having a previous foot ulcer and location of the previous foot ulcer. The area under the receiver operating curve was 0.76 (IQR $0.66-0.87$ ) and the Brier score was 0.17 (IQR 0.15-0.18).

Conclusions These validated prediction models help identify those patients that are at increased risk of plantar foot ulcer recurrence and for that reason should be monitored more carefully and treated more intensively.

\section{INTRODUCTION}

Foot ulceration is a common and feared complication in people with diabetes mellitus; its presence has a great impact on the individuals' quality of life, healthcare and society. ${ }^{12}$ The annual incidence of a foot ulcer in people with diabetes is approximately $2 \% .^{3}$ The risk of developing an ulcer increases if peripheral neuropathy, a history of ulceration, a foot deformity and/or peripheral vascular disease is present. ${ }^{4-6}$ Approximately $40 \%$ of patients who heal from an ulcer have a recurrence in the first 12 months and $60 \%$ within 3 years. ${ }^{7}$

\section{Significance of this study}

What is already known about this subject?

- Several etiological risk factors models exist to identify variables that are associated with ulcer recurrence in high-risk people with diabetes; most risk factors are non-modifiable.

- However, most studies use inconsistent terminology to describe these models and to interpret them and are not validated.

- A validated prediction model allows more accurate assessment of ulcer recurrence risk and provides valuable information for patient follow-up and treatment.

What are the new findings?

- The presence of a minor lesion, living alone, increased barefoot peak plantar pressure, longer duration of having a previous foot ulcer and less variation in daily stride count are predictors of plantar foot ulcer recurrence in high-risk people with diabetes.

- Predictors of plantar foot ulcer recurrence attributed to unrecognized repetitive stress as primary biomechanical mechanism are presence of a minor lesion, longer duration of having a previous foot ulcer and location of the previous foot ulcer.

- Most predictors are variables that can be easily obtained by healthcare professionals and some predictors are modifiable factors that can be targeted for intervention.

How might these results change the focus of research or clinical practice?

- These prediction models allow the clinician and practitioner to timely identify patients who are at risk of developing a recurrent plantar foot ulcer and to communicate this risk with the patient.

- Additionally, they can be used to select suitable patients for therapy and guide clinician and patient in joint decision-making for preventative treatment.

This high recurrence rate is due to the many contributing factors that are still present after healing of the first ulcer, such as, neuropathy, foot deformity, increased plantar stress and peripheral vascular disease. Because of 
the high incidence of recurrence and subsequent risk of infection, hospital admission and amputation, a strong focus in diabetic foot disease is currently on the "patient in remission' and prevention of foot ulcer recurrence. ${ }^{7}$ To develop adequate strategies for prevention, it is important to identify predictors of foot ulcer recurrence in diabetes.

The risk factors for diabetic foot ulcer recurrence have recently been reviewed by Armstrong et al. ${ }^{7}$ The strongest independent risk factors reported were: a vibration perception threshold $>25 \mathrm{~V},{ }^{8}$ the presence of minor lesions (eg, abundant callus, blister formation or hemorrhage) ${ }^{9}$ the plantar location of the previous ulcer ${ }^{10} 11$ and the presence of peripheral vascular disease..$^{10}$ Many risk factor models have been developed with various clinical outcomes in mind, such as ulcer recurrence. ${ }^{2}$ 8-12 However these studies are inconsistent in description and interpretation of these models, use different starting points for patient follow-up, identify only individual etiological risk factors and are often not validated. A validated prediction model uses multiple variables to more accurately predict the risk of a future outcome, regardless of causality between the predictor and outcome. ${ }^{13}$

Well-designed prediction models can be of additional value in the prevention of ulcer recurrence. A prediction model allows the clinician or practitioner to timely identify patients that are at risk of developing a recurrent foot ulcer and to communicate this risk with the patient. Additionally, it can be used to select suitable patients for therapy and guides the clinician and patients in joint decision-making for preventative treatment. This applies, for example, to the frequency at which high-risk patients are screened to help identity risk and to prevent foot ulceration (once every $1-3$ months is currently the recommendation for high-risk patients in international guidelines). ${ }^{14}$ Therefore, the aim of this study was to develop a prediction model for plantar foot ulcer recurrence in high-risk people with diabetes and to validate its predictive performance.

\section{METHODS}

Population

Data were retrieved from a multicenter randomized controlled trial on effectiveness of custom-made footwear to prevent plantar foot ulcer recurrence. ${ }^{15}$ Patients were recruited between 2007 and 2010 from the multidisciplinary outpatient diabetic foot clinics of two academic and eight large general public hospitals across the Netherlands. From a total 267 possibly eligible participants, 171 people with diabetes with loss of protective sensation, a recent history of plantar foot ulceration $(<18$ months prior to inclusion) and newly prescribed custom-made footwear were included in this study. Loss of protective sensation was assessed using $10 \mathrm{~g}$ Semmes-Weinstein monofilament and biothesiometer (Biomedical Instruments, Newbury, Ohio, USA) testing. ${ }^{16}$ Patients were excluded if they had an active plantar ulcer, bilateral amputation proximal to the tarso-metatarsal (Lisfranc) joint, an estimated survival of $<18$ months and the inability to walk unaided. Participants were randomly assigned to pressure-improved custom-made footwear ( $20 \%$ peak pressure relief by modifying the footwear) or non-improved custom-made footwear. Follow-up time was 18 months or until plantar foot ulceration. Written informed consent was obtained prior to inclusion from all patients.

\section{Potential predictors}

As potential predictors of plantar foot ulcer recurrence, ${ }^{9}{ }^{15}$ demographic, disease-related, biomechanical and behavioral factors were included.

The demographic and disease-related factors were collected at baseline through anamnesis or physical examination and included: age, gender, body mass index, diabetes type and duration, glycated hemoglobin (HbA1c), smoking (history), alcohol consumption, living alone, employment status, highest education level, vibration perception threshold, presence of peripheral arterial disease (grade $\mathrm{I}$ or $\mathrm{II}^{17}$ ), duration of previous ulcer(s), time between healing of the previous ulcer and study entry, location of the previous ulcer (ie, hallux, second to fifth toe, metatarsal heads or midfoot), history of amputation, severity of foot deformity and the presence of minor lesions. Foot deformity was defined as absent, mild, moderate, severe and major amputations. ${ }^{915}$ Minor lesions were defined as non-ulcerative lesions of the skin on the plantar foot, including abundant callus, hemorrhage or a blister.

The biomechanical variables assessed at study entry were barefoot plantar foot pressure (measured using an Emed-X pressure platform, Novel, Munich, Germany) and in-shoe plantar foot pressures (measured using a Pedar-X system, Novel) during comfortable level walking. Regional peak barefoot and in-shoe plantar pressure were calculated as well as two parameters that represented the cumulative load on the foot: weighted pressure (WP) and cumulative plantar tissue stress (CPTS), as described elsewhere. $^{915}$

The behavioral factors assessed during the study were footwear adherence and walking activity. Adherence to wearing prescribed footwear was measured over a 7-day period using the @monitor (Academic Medical Center, Amsterdam, the Netherlands ${ }^{18}$ ). Next to overall adherence, adherence was assessed for when patients were at home and when away from home, using self-report forms. Walking activity was measured as stride count over the same 7-day period, using a StepWatch activity monitor (Orthocare Innovations, Oklahoma City, Oklahoma, USA). ${ }^{915}$ The outcome parameters were average daily stride count and day-to-day variation in stride count (ie, SD in daily stride count over a 7-day period).

For the parameters footwear adherence at home and away from home, $>25 \%$ of the data were missing across subjects (namely 39.2\%), and these parameters were therefore excluded as potential predictor. We used 
multivariate imputations for parameters with up to $25 \%$ of missing data by applying the chained equations (mice) approach as implemented by the mice package in $\mathrm{R} .{ }^{19}$ This provided multiple imputations for multivariate missing data regardless of variable type, where each incomplete variable is imputed by a separate model (this is the fully conditional specification method). We used five imputation sets with a maximum of two iterations and the quick selection of predictor option, which is useful when there are many variables. Little's missing completely at random test $^{20}$ failed to show potential patterns in missing data $\left(\chi^{2}=58.57, \mathrm{df}=49, \mathrm{p}=0.16\right)$.

\section{Primary outcome}

The primary outcome was plantar foot ulcer recurrence in 18 months. Foot ulcer was defined as a full-thickness lesion of the skin, irrespective of duration. ${ }^{14}{ }^{17}$ Recurrence was defined as an ulcer at the same location as the previous one, or at any other plantar location on the ipsilateral or contralateral foot. If a patient, treating physician, or other healthcare provider (eg, podiatrist) identified an ulcer during follow-up, they were instructed to report the lesion, complete a foot ulcer form and have photographs of the lesion taken. During 3-monthly follow-up visits, patients were asked about any lesion that had occurred and electronic patients files were checked for any unreported ulcer. Outcome assessment was done blinded by three independent diabetic foot experts who assessed photographs of the plantar foot if an ulcer was suspected. Two additional foot experts were consulted when unanimity was not reached.

\section{Model development}

Two logistic regression prediction models of plantar foot ulcer recurrence were developed. The first model was on prediction of all recurrent plantar foot ulcers in the study. The second model was on prediction of those recurrent plantar foot ulcers that were suggested to be the result of unrecognized repetitive stress. This was defined as an ulcer occurring at the same location as the previous ulcer and not being the result of a traumatic event, as reported by the patient. This division in models was analogous to the study by Waaijman et al..$^{9}$ Dependent on the prediction model, the foot with the worst outcome for a given parameter with bilateral outcomes was chosen (first model), or the foot where the previous ulcer was located (second model). Reporting on the development of these models was done according to the Transparent Reporting of a multivariable prediction model for Individual Prognosis Or Diagnosis statement. ${ }^{21}$

Based on clinical reasoning, knowledge from the literature and clinical feasibility in assessment, we considered all the above-mentioned potential predictors as variables in the model. Potential predictors that showed to be strongly correlated with each other (ie, correlation coefficient $>0.5$ ) contribute little independent information to the model. Using clinical reasoning regarding which potential factor to exclude, we excluded the following variables based on high intervariable correlation: age, HbAlc and type of diabetes (all correlated with duration of diabetes), education (correlated with living alone) and average daily stride count (correlated with day-to-day variation in stride count). Both WP and CPTS were excluded from the model, because they strongly correlated with each underlying factor in these composite variables.

\section{Model fitting and validation}

The model development strategy went through four stages: (a) creating five imputed datasets with no missing values, (b) further variable selection in each imputed dataset, (c) fitting a logistic regression model on each of the five imputed datasets to predict ulcer outcome based on these variables and (d) pooling these five models into a final prediction model. The final logistic regression model for plantar foot ulcer recurrence will be represented by its linear predictor (LP). The predicted probability can be calculated from this LP with the following formula: $1 /\left(1+e^{-\mathrm{LP}}\right)$.

Further variable selection (stage 'b' above) was deemed important because after initial expert selection of variables many potential predictors remained and the dataset of 171 patients is relatively small. Definitive variables for the model were selected in two steps. First, we selected variables that had a univariable association with the primary outcome with a $\mathrm{p}$ value $<0.2$. Second, we developed a multivariable model with those selected variables and used backward variable selection based on the Akaike Information Criterion (AIC) ${ }^{22} 23$ aiming at finding the optimal set of predictors. By giving a penalty for model complexity (in terms of the number of included variables), the AIC strikes a good balance between the likelihood of the model (which always increases with the number of included variables) and its complexity (the more complex the model, the more likely it would overfit the data).

We used 10-fold cross-validation to internally validate the prediction strategy. This means that the whole model development strategy (including the five multiple imputation datasets and the variable selection process) is repeated in each of the 10 folds on the training set $(90 \%$ of the data) and tested on the $10 \%$ held-out dataset of that fold.

\section{Model performance}

Model performance was assessed in terms of discrimination and calibration. ${ }^{24}$ Discrimination was measured for all five pooled models and the final prediction model by the area under the receiver operating curve (AUC) using the median, IQR and minimum and maximum over 10 folds. ${ }^{25}$ The AUC curves of the final models are also presented. It refers to the ability of the model to provide a higher probability of the event (ie, ulcer recurrence) to those patients with the event than those without the event. The higher the value of the AUC the better the discrimination ability. Calibration refers to the closeness of the predicted probabilities to the true ones as estimated 


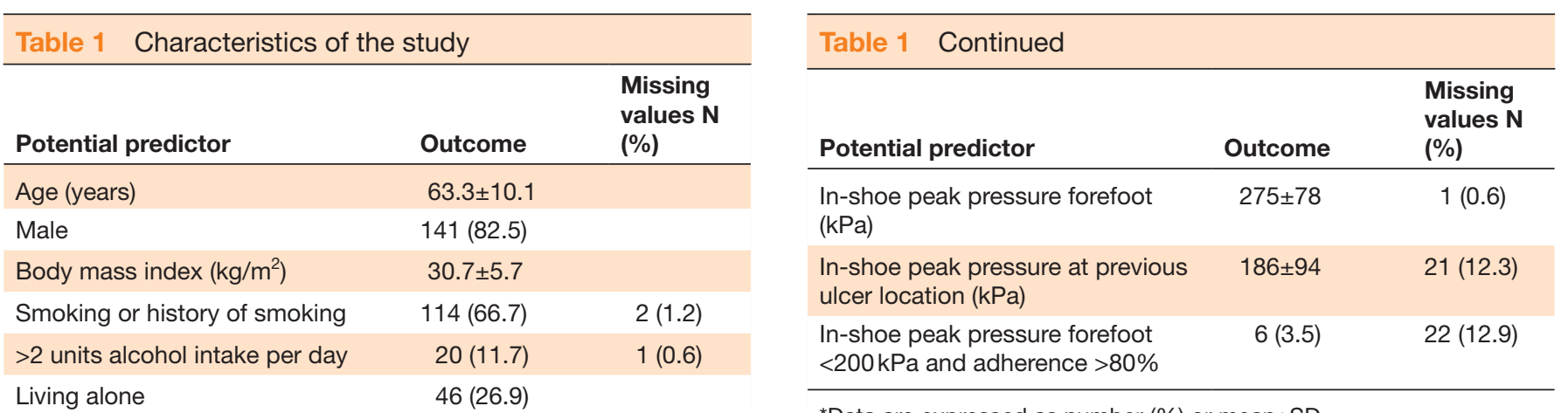

${ }^{*}$ Data are expressed as number (\%) or mean \pm SD.

by appropriate patient groups, and was assessed using calibration graphs. The Brier score,${ }^{26}$ which is the mean squared error of a prediction, combines both elements of discrimination and calibration and was also assessed for all five models on the imputed datasets and the final pooled prediction model (median, IQR and minimum and maximum over 10 folds). A Brier score ranges from 0 to 1 and if the predicted values by the model and the observed values are completely concordant then the Brier score is 0 . Finally, the positive predictive value (PPV), the proportion of positive results that are truly positive, was calculated in each fold when the threshold was set at the 75th percentile of predictions.

We used the average predictive comparison to assess the change on the probability of the outcome due to the change in each predictor in the model, hence indicating the influence of each of the individual predictors on the probability of ulcer recurrence when all other predictors remain constant. ${ }^{27}$ Descriptive statistics were performed using SPSS V.22.0 software (IBM, Armonk, New York, USA). All model analyses were performed in the $\mathrm{R}$ statistical environment ( $\mathrm{R}$ Foundation for Statistical Computing for Windows V.2.9.0 (http://www.Rproject.org) ). ${ }^{28}$

\section{RESULTS}

Table 1 describes the characteristics of the study sample. Of the total 171 patients, 141 were male and the mean age was 63.3 years. Seventy-one patients $(=42 \%)$ had a recurrent ulcer with a mean time to ulceration of 197 days. Forty-one of those 71 patients $(=24 \%$ of the total group) had a recurrent ulcer due to unrecognized repetitive stress, with a mean time to ulceration of 173 days.

\section{Model 1: all recurrent plantar foot ulcers}

The model for this outcome contained five predictors (table 2): increased barefoot peak plantar pressure at the forefoot (in $\mathrm{kPa}$ ), presence of a minor lesion, duration of the previous ulcer in months and living alone were positive predictors for recurrence; a higher variation in day-to-day stride count (in SDs) was a negative predictor of recurrence.

The LP of the logistic regression model for recurrent plantar foot ulcer was: $-2.1+0.76 \times$ living alone $+1.4 \times$ minor lesion present $+0.034 \times$ duration of previous ulcer in 
Table 2 Predictors for model 1 (all recurrent plantar foot ulcer) and model 2 (plantar foot ulcer recurrence from unrecognized repetitive stress)

\begin{tabular}{|c|c|c|c|c|}
\hline Predictor & Coefficient & $95 \% \mathrm{Cl}$ & Change in variable & $\begin{array}{l}\text { Change } \\
\text { in ulcer } \\
\text { probability }\end{array}$ \\
\hline \multicolumn{5}{|c|}{ Model 1: all plantar foot ulcer recurrences } \\
\hline Intercept & -2.1 & -3.8 to -0.37 & & \\
\hline Living alone & 0.76 & 0.015 to 1.5 & No to yes & 0.16 \\
\hline Minor lesions & 1.4 & 0.69 to 2.1 & No to yes & 0.25 \\
\hline Duration of the previous ulcer & 0.034 & 0.0026 to 0.065 & 12 months & 0.085 \\
\hline Barefoot peak plantar pressure & 0.0013 & -0.00013 to 0.0027 & $255 \mathrm{kPa}$ & 0.07 \\
\hline Variation in daily stride count & -0.047 & -0.10 to 0.0098 & 700 steps & -0.065 \\
\hline \multicolumn{5}{|c|}{ Model 2: plantar foot ulcer recurrence from unrecognized repetitive stress } \\
\hline Intercept & -1.8 & -2.5 to -1.1 & & \\
\hline Minor lesions & 2.2 & 1.3 to 3.1 & No to yes & 0.37 \\
\hline Duration of the previous ulcer & 0.038 & 0.0047 to 0.071 & 12 months & 0.064 \\
\hline \multicolumn{5}{|l|}{ Previous ulcer location: } \\
\hline Metatarsal heads & Reference & & & \\
\hline Hallux & -1.6 & -2.8 to -0.4 & \multirow{3}{*}{$\begin{array}{l}\text { In comparison to } \\
\text { patients with an ulcer at } \\
\text { the metatarsal heads }\end{array}$} & -0.028 \\
\hline Toes & -2.0 & -3.6 to -0.4 & & -0.21 \\
\hline Midfoot & 0.024 & -2.3 to 2.4 & & 0.21 \\
\hline
\end{tabular}

$\mathrm{Cl}$, Confidence interval.

months $+0.0013 \times$ barefoot peak plantar pressure at the forefoot in $\mathrm{kPa}-0.047 \times$ variation in daily stride count in SDs.
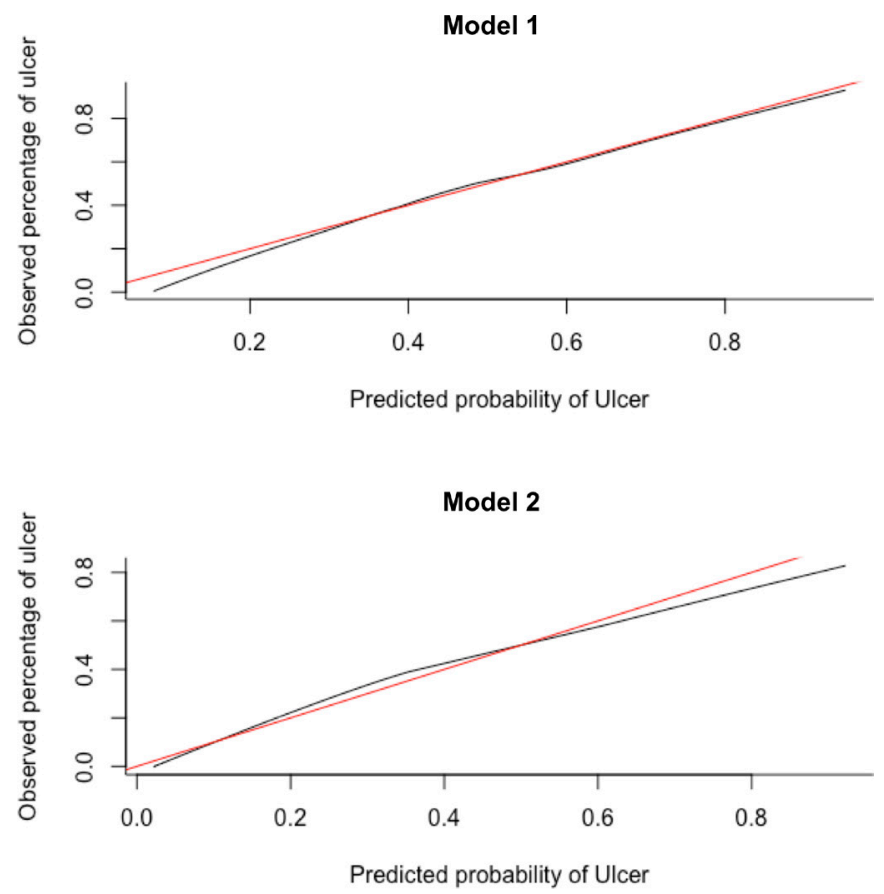

Figure 1 Calibration graphs for model 1 (all recurrent plantar foot ulcer) and model 2 (plantar foot ulcer recurrence from unrecognized repetitive stress). In each graph the black line shows the observed proportion of the event versus the probability of the event as predicted by the model. Ideally all the points fall on the diagonal red line.
Table 2 also shows the average predictive comparison for model 1. If a patient has a minor lesion present or lives alone there is a 0.25 and 0.16 higher probability, respectively, for ulcer recurrence. If a patient has a duration of past ulceration of 12 months or an increase in barefoot peak plantar pressure of $255 \mathrm{kPa}$, there is a higher probability of 0.085 or 0.07 , respectively, for ulcer recurrence. An increase of variation in day-to-day stride count of 700 steps decreases the probability for ulcer recurrence with -0.065 .

Figure 1 shows the calibration graph based on the average predictions per patient of the final model on the five imputation datasets. The graph shows that the predicted probability of a recurrent ulcer and the observed number of recurrent ulcers agreed over almost the whole range of probabilities. Only when the predicted probability is lower than 0.35 , the prediction slightly underestimates the proportion of observed recurrent ulcers. Figure 2 shows the AUC of the final model. The median AUC of this final model was 0.68 (IQR 0.61-0.80). The minimum AUC was 0.53 and the maximum AUC was 0.89 over the 10 folds with a SD of 0.159. The median Brier score was 0.24 (IQR 0.20-0.28). The median PPV was 65\% (IQR 50\%-79\%).

\section{Model 2: plantar foot ulcer recurrence from unrecognized repetitive stress}

The model for this outcome contained three predictors (table 2): presence of a minor lesion, duration of the previous ulcer in months and the location of the previous ulcer. 

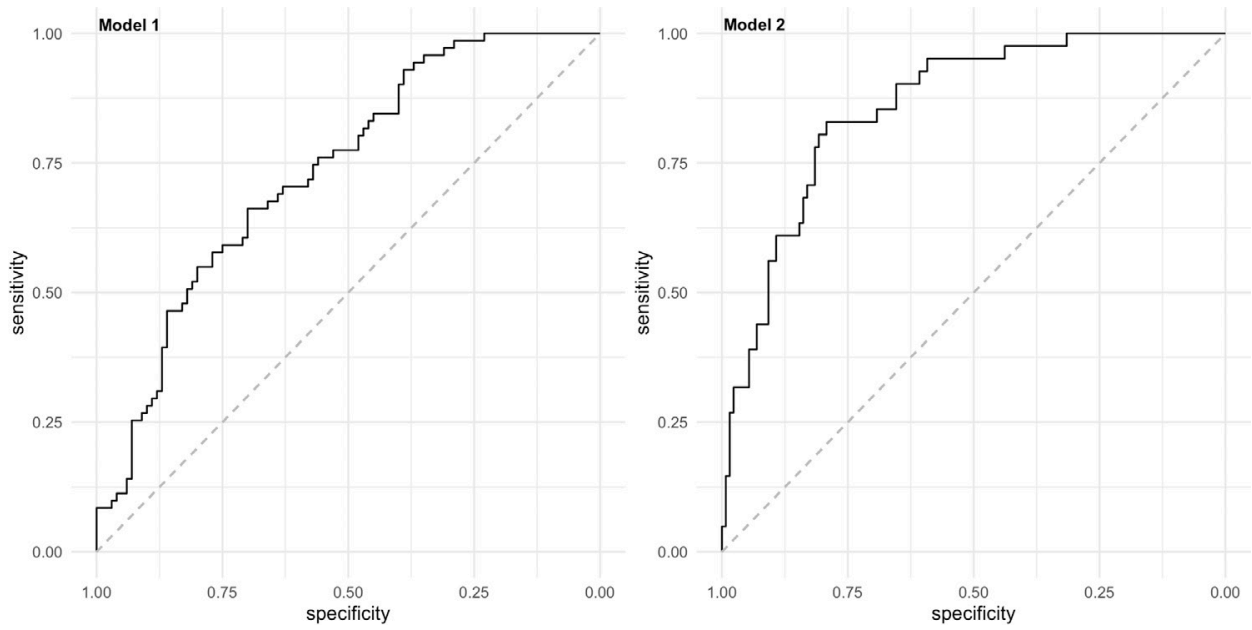

Figure 2 Area under the receiver operating curve for model 1 (all recurrent plantar foot ulcer) and model 2 (plantar foot ulcer recurrence from unrecognized repetitive stress).

Based on these results, the LP of the logistic regression model was: $-1.8+2.2 \times$ minor lesion present $+0.038 \times \mathrm{du}-$ ration of previous ulcer in months+-1.6×ulcer location 'hallux'; -2.0×ulcer location 'lesser toes'; $0.024 \times$ ulcer location 'midfoot'. For this formula, ulcer location under the metatarsal heads was the reference category.

The average predictive comparison is shown in table 2. If a patient has a minor lesion or a duration of past ulceration of 12 months, there is a 0.37 and 0.064 higher probability, respectively, for ulcer recurrence. If the previous ulcer was located on the plantar hallux or toes, the probability for ulcer recurrence decreased with -0.028 and -0.21 , respectively compared with patients who had the previous ulcer under the metatarsal heads. However, if the previous ulcer was located under the midfoot, the probability increased with 0.21 compared with patients with a previous ulcer under the metatarsal heads.

Figure 1 shows the calibration graph based on the average predictions per patient for the model on the five imputation datasets. The graph shows that the predicted probability of ulcer recurrence from unrecognized repetitive stress slightly overestimates the observed proportion of recurrent ulcers from unrecognized repetitive stress when the predicted probability is between 0.10 and 0.50 and slightly underestimates the observed proportion of recurrent ulcers from unrecognized repetitive stress when the predicted probability is higher than 0.50 . Figure 2 shows the AUC of the final model. The median AUC of this final model was 0.76 (IQR 0.66-0.87). The minimum AUC was 0.50 and the maximum AUC was 0.88 over 10 folds with a SD of 0.175 . The median Brier score was 0.17 (IQR 0.15-0.18). The median PPV was $65 \%$ (IQR 50\%-79\%).

\section{DISCUSSION}

This study showed that presence of a minor lesion, living alone, increased barefoot peak plantar pressure, longer duration of having a previous foot ulcer and less variation in daily stride count are predictors of plantar foot ulcer recurrence in high-risk people with diabetes. The prediction model showed relatively poor discrimination but had good calibration. Presence of a minor lesion and longer duration of having a previous foot ulcer were also predictors of plantar foot ulcer recurrence attributed to unrecognized repetitive stress, in addition to location of the previous foot ulcer. The model showed fair discrimination and reasonable calibration.

The first prediction model contains a combination of biomechanical, behavioral, patient-related and disease-related factors; the second model only includes biomechanical and disease-related factors. The fact that both models include biomechanically related factors is because we focus on foot ulcers on the plantar surface, which have a stronger biomechanical etiology than nonplantar foot ulcers. ${ }^{7}$ The presence of a minor lesion was in both models a predictor, showing the largest observed change in ulcer recurrence probability of all predictors. This is in accordance with the study by Waaijman et al, who showed on the same data set that presence of a minor lesion was the strongest associated factor with plantar ulcer recurrence. ${ }^{9}$ Minor lesions such as abundant callus and blisters are the result of mechanical stress and are therefore amendable through pressure-relieving footwear. Furthermore, they allow early identification of impending ulceration that helps to inform the patient about risk and helps to reduce ulcer recurrence risk if treated appropriately. ${ }^{15} 29$

Living alone predicts plantar foot ulcer recurrence in our first model. This suggest that partners or relatives are important in helping to preserve the patient's foot health. Social status and its association with ulcer recurrence was previously investigated, but has not before shown to be a significant one ${ }^{930}$ Variation in stride count negatively predicted ulcer recurrence in the first model, suggesting that less variation in daily stride count predicts recurrence. This is contrary to the study by Armstrong $e t$ $a l$, who found in medium-risk to high-risk patients that a higher variability in daily stride count increases risk of 
ulceration. ${ }^{31}$ They postulate that high-risk patients are less able to withstand repetitive stress and that modulating the 'peaks and valleys' of their daily stride activity might reduce ulcer recurrence risk. ${ }^{31}$ These authors also showed that daily stride count in patients who ulcerated was significantly lower than in those who did not, an outcome that was not found in our data. ${ }^{9}$ This sounds counterintuitive given the lower cumulative stress exerted on the foot in these non-ulcerated cases, but suggestions that biomechanical loading of the foot leads to tissue adaptation and improved load tolerance, ${ }^{32}{ }^{33}$ supports these findings. More research is needed to untangle the apparent complex interaction between amount of daily activity and risk of plantar foot ulcer recurrence.

The location of the previous foot ulcer predicted recurrence in our second model. The probability of developing an ulcer at the same location was lower for a previous ulcer at the hallux compared with one at the metatarsal heads, and even lower for a previous ulcer at the lesser toes. The probability of ulcer recurrence at the midfoot was high, likely because all patients with a midfoot ulcer had Charcot midfoot deformity. In general, a plantar location of a previous ulcer increases risk of ulcer recurrence. ${ }^{911}$ Peters et al found that plantar hallux ulcers are more prone to recurrence than any other ulcer (plantar or dorsum). ${ }^{10}$ The distribution of plantar pressures over the foot likely explains our results, where highest pressures are generally found at the metatarsal heads, followed by the hallux and then the lesser toes. ${ }^{34}$ Offloading these high-risk areas can help in reducing ulcer recurrence risk. ${ }^{1535}$

Most predictors identified in both models are variables that can be easily and readily obtained by healthcare professionals through anamnesis, physical examination and measurement. Only barefoot plantar pressure analysis is not easily obtained in every setting, although its use is increasing, and the need for such measurements is indicated in this and other studies. For the purpose of clinical practice, it is possible to integrate these models in an electronic healthcare system that can provide predictive risk when data input based on anamnesis and physical examination is completed. When using both models, the treating physician should be aware that the first model slightly underestimates the risk in patients at a low risk of ulcer recurrence, while the second model slightly overestimates the patients at low risk of ulcer recurrence and slightly underestimates the patients at high risk of ulcer recurrence. Based on the second model, it might therefore be possible that patients with a high predicted probability of ulcer recurrence may be treated or seen less frequently than they supposed to be based on the actual probability of ulcer recurrence.

However, while accurate predictions give valuable insight into which patients are at a high risk of developing plantar foot ulcer recurrence and need more frequent follow-up, the coefficients in our prediction models are mainly useful for implementing these models by others (eg, for external validation). They should not be interpreted causally, and due to possible correlations between them odds ratios might not be meaningful. Nevertheless, some predictors are modifiable factors that can be targeted for intervention using current literature and clinical knowledge. Minor lesions, for example, can be treated on sight and peak plantar pressures can be reduced by limiting barefoot walking. ${ }^{35}$ Advice regarding an appropriate and safe level of daily activity is also possible. ${ }^{35}$ It is important, however, to stress that it is unclear what effect these interventions will have on the predicted risk of plantar foot ulcer recurrence.

Several strengths and limitations apply to this study. We used the same dataset as Waaijman et al; however, their models are etiological in nature and aim to explain whether an ulcer recurrence can reliably be attributed to a risk factor. Missing data were not accounted for by these authors, which may lead to bias. In our models, missing data were multiply imputed. Additionally, their study lacked internal validation; the reported sensitivity of $81 \%$ and specificity of $50 \%$ are likely overestimated. Another strength of our study is that we used AIC and cross-validation for the selection of potential predictors while other studies used a multivariate regression analysis with significant factors $(p<0.10)$ from a univariate analysis. ${ }^{2}{ }^{8-11}$ Also, most studies do not or only partly report the performance of their models in terms of discrimination and calibration. ${ }^{28-11}$

A first and important limitation is the limited number of patients included in our database. With only 71 and 41 events for model 1 and 2, respectively, only a small number of predictors is warranted in the model in order to avoid overfitting. Because we have many candidate predictors, the choice of predictor set is not very stable and other predictors could be selected when having other samples of the same size. However, we relied on clinical knowledge for the initial selection of variables, then we used a liberal $p$ value of 0.2 for the second stage and then used the AIC to select the remaining variables. Second, the outcome of the second model was partly based on the patient's self-report that an ulcer was not a result of an acute trauma, which might introduce a recall bias. Third, some variables had too much missing data that prevented us from including them in the model. Finally, external validation of our model on another database to evaluate model performance in other high-risk patients with diabetes was not performed.

\section{CONCLUSION}

We provided well-designed and internally validated prediction models for risk of plantar foot ulcer recurrence in high-risk people with diabetes. The model predicted recurrence based on presence of a minor lesion, living alone, increased barefoot peak plantar pressure, longer duration of having a previous foot ulcer and less variation in daily stride count, with good calibration but relatively poor discrimination. The model for repetitive stress ulcers predicted recurrence based on presence 
of a minor lesion, longer duration of having a previous foot ulcer and the location of the previous ulcer, with fair discrimination and a reasonable calibration. These models help identify those patients that are at increased risk of plantar foot ulcer recurrence and for that reason should be monitored more carefully and frequently and treated more intensively.

Contributors WBadS, AA-H, SAB conceived and designed the analysis for this study, using existing data. $A A-H$ performed the statistical analysis. WBadS wrote the manuscript. AA-H and SAB critically reviewed and edited the manuscript. All authors have read and approved the final manuscript.

Funding The authors have not declared a specific grant for this research from any funding agency in the public, commercial or not-for-profit sectors.

Competing interests None declared.

Patient consent for publication Not required.

Ethics approval The research ethics committee of all 10 participating centers in the trial approved this study.

Provenance and peer review Not commissioned; externally peer reviewed.

Data availability statement Data are available on reasonable request.

Open access This is an open access article distributed in accordance with the Creative Commons Attribution 4.0 Unported (CC BY 4.0) license, which permits others to copy, redistribute, remix, transform and build upon this work for any purpose, provided the original work is properly cited, a link to the licence is given, and indication of whether changes were made. See: https://creativecommons.org/ licenses/by/4.0/.

ORCID iDs

Wouter B aan de Stegge http://orcid.org/0000-0003-4834-1691

Sicco A Bus http://orcid.org/0000-0002-8357-9163

\section{REFERENCES}

1 Kerr M, Rayman G, Jeffcoate WJ. Cost of diabetic foot disease to the National health service in England. Diabet Med 2014:31:1498-504.

2 Gonzalez JS, Vileikyte L, Ulbrecht JS, et al. Depression predicts first but not recurrent diabetic foot ulcers. Diabetologia 2010;53:2241-8.

3 Crawford F, McCowan C, Dimitrov BD, et al. The risk of foot ulceration in people with diabetes screened in community settings: findings from a cohort study. QJM 2011;104:403-10.

4 Abbott CA, Carrington AL, Ashe H, et al. The north-west diabetes foot care study: incidence of, and risk factors for, new diabetic foot ulceration in a community-based patient cohort. Diabet Med 2002;19:377-84.

5 Boulton AJM, Vileikyte L, Ragnarson-Tennvall G, et al. The global burden of diabetic foot disease. Lancet 2005;366:1719-24.

6 Boyko EJ, Ahroni JH, Stensel V, et al. A prospective study for risk factors for diabetic foot ulcer. Diabetes Care 1999;22:1036-42.

7 Armstrong DG, Boulton AJM, Bus SA. Diabetic foot ulcers and their recurrence. N Engl J Med 2017;376:2367-75.

8 Monami M, Longo R, Desideri CM, et al. The diabetic person beyond a foot ulcer: healing, recurrence, and depressive symptoms. J Am Podiatr Med Assoc 2008;98:130-6.

9 Waaijman R, de Haart M, Arts MLJ, et al. Risk factors for plantar foot ulcer recurrence in neuropathic diabetic patients. Diabetes Care 2014;37:1697-705.

10 Peters EJG, Armstrong DG, Lavery LA. Risk factors for recurrent diabetic foot ulcers: site matters. Diabetes Care 2007;30:2077-9.

11 Dubský M, Jirkovská A, Bem R, et al. Risk factors for recurrence of diabetic foot ulcers: prospective follow-up analysis in the Eurodiale subgroup. Int Wound J 2013;10:555-61.
12 Monteiro-Soares M, Boyko EJ, Ribeiro J, et al. Predictive factors for diabetic foot ulceration: a systematic review. Diabetes Metab Res Rev 2012;28:574-600.

13 Moons KGM, Royston P, Vergouwe Y, et al. Prognosis and prognostic research: what, why, and how? BMJ 2008;388.

14 Schaper NC, Van Netten JJ, Apelqvist J, et al. Prevention and management of foot problems in diabetes: a summary guidance for daily practice 2015 , based on the IWGDF guidance documents. Diabetes Metab Res Rev 2016;32 Suppl 1:7-15.

15 Bus SA, Waaijman R, Arts ML, et al. Effect of custom-made footwear on foot ulcer recurrence in diabetes. Diabetes Care 2013;36:4109-16.

16 Pham $\mathrm{H}$, Armstrong DG, Harvey $\mathrm{C}$, et al. Screening techniques to identify people at high risk for diabetic foot ulceration: a prospective multicenter trial. Diabetes Care 2000;23:606-11.

17 Schaper NC. Diabetic foot ulcer classification system for research purposes: a progress report on criteria for including patients in research studies. Diabetes Metab Res Rev 2004;20 Suppl $1:$ S90-5.

18 Bus SA, Waaijman R, Nollet F. New monitoring technology to objectively assess adherence to prescribed footwear and assistive devices during ambulatory activity. Arch Phys Med Rehabil 2012:93:2075-9.

19 Van Buuren S, Groothuis-Oudshoorn K. Mice: multivariate imputation by chaned equations in R. J Stat Softw 2011;45:1-67.

20 Little RJA. A test of missing completely at random for multivariate data with missing values. J Am Stat Assoc 1988;83:1198-202.

21 Collins GS, Reitsma JB, Altman DG, et al. Transparent reporting of a multivariable prediction model for individual prognosis or diagnosis (TRIPOD): the TRIPOD statement. Br J Surg 2015;102:148-58.

22 Akaike $\mathrm{H}$. Information theory as an extension of the maximum likelihood principle. In: Petrov BN, Csaki F, eds. Second International Sympposium on information theory; Akademiai Kiado. Budapest, 1973: 267-81.

23 Sauerbrei W. The use of resampling methods to simplify regression models in medical statistics. J R Statist Soc C 1999;48:313-29.

24 Harrell FE, Lee KL, Mark DB. Multivariable prognostic models: issues in developing models, evaluating assumptions and adequacy, and measuring and reducing errors. Stat Med 1996;15:361-87.

25 Hanley JA, McNeil BJ. The meaning and use of the area under a receiver operating characteristic $(\mathrm{ROC})$ curve. Radiology 1982;143:29-36.

26 Brier GW. Verification of forecasts expressed in terms of probability. Monthly Weather Review 1950;78:1-3.

27 Hanushek EA, Jackson JE. Statistical methods for social scientists. New York: Academic Press, 1977.

28 R-Core-Team. A language and environment for statistical computing. Vienna, Austria, 2019. https://www.r-project.org

29 van Netten JJ, Price PE, Lavery LA, et al. Prevention of foot ulcers in the at-risk patient with diabetes: a systematic review. Diabetes Metab Res Rev 2016;32 Suppl 1:84-98.

30 Kloos C, Hagen F, Lindloh C, et al. Cognitive function is not associated with recurrent foot ulcers in patients with diabetes and neuropathy. Diabetes Care 2009;32:894-6.

31 Armstrong DG, Lavery LA, Holtz-Neiderer K, et al. Variability in activity may precede diabetic foot ulceration. Diabetes Care 2004;27:1980-4.

32 Maluf KS, Mueller MJ. Comparison of physical activity and cumulative plantar tissue stress among subjects with and without diabetes mellitus and a history of recurrent plantar ulcers. Clin Biomech 2003;18:567-75.

33 Mueller MJ, Maluf KS. Tissue adaptation to physical stress: a proposed "Physical Stress Theory" to guide physical therapist practice, education, and research. Phys Ther 2002;82:383-403.

34 Barn R, Waaijman R, Nollet F, et al. Predictors of barefoot plantar pressure during walking in patients with diabetes, peripheral neuropathy and a history of ulceration. PLoS One 2015;10:e0117443.

35 Bus SA, van Netten JJ, Lavery LA, et al. IWGDF guidance on the prevention of foot ulcers in at-risk patients with diabetes. Diabetes Metab Res Rev 2016;32 Suppl 1:16-24. 